\title{
Effects of Anodal Transcranial Direct Current Stimulation and Serotonergic Enhancement on Memory Performance in Young and Older Adults
}

\author{
Kristin Prehn", , Helena Stengl', Ulrike Grittner ${ }^{2,3}$, René Kosiolek', Anja Ölschläger', Alexandra Weidemann' \\ and Agnes Flöel ${ }^{*, 1,3}$ \\ 'Department of Neurology and NeuroCure Clinical Research Center, Charité Universitätsmedizin Berlin, Berlin, Germany; \\ ${ }^{2}$ Department of Biostatistics and Clinical Epidemiology, Charité Universitätsmedizin Berlin, Berlin, Germany; ${ }^{3}$ Center for Stroke Research, \\ Charité Universitätsmedizin Berlin, Berlin, Germany
}

\begin{abstract}
In the absence of effective therapies for dementia and its precursors, enhancing neuroplasticity by means of non-invasive brain stimulation such as anodal transcranial direct current stimulation (atDCS) might be a promising approach to counteract or delay the onset of cognitive decline, but effect sizes have been moderate so far. Previous reports indicate that increasing serotonin levels may enhance atDCS-induced neuroplasticity. However, evidence for serotonergic modulation of atDCS effects on memory is still lacking. Here, we conducted a doubleblind, randomized, sham-/placebo-controlled trial to investigate the impact of a selective serotonin reuptake inhibitor (SSRI; single dose of $20 \mathrm{mg}$ citalopram) and atDCS over the right temporoparietal cortex (I mA, 20 min) on memory formation. Twenty young and 20 older subjects completed an object-location learning task in each of the four conditions: sham+placebo, sham+SSRI, atDCS+placebo, and atDCS+SSRI. Outcome measures were performance in immediate (primary outcome) and delayed cued recall. While we found an SSRI effect, but no statistically significant effect of atDCS on immediate recall scores, young and older adults benefited most from the combined application (comparisons: atDCS+SSRI>atDCS+placebo and atDCS+SSRI>sham+placebo). Thus, our data provide evidence that atDCS improves memory formation if serotonergic neurotransmission is enhanced simultaneously. Further studies are needed to assess whether these findings extend to clinical populations with memory impairment and translate into clinically relevant improvements after long-term serotonergic enhancement and repeated stimulation.
\end{abstract}

Neuropsychopharmacology (2017) 42, 55 I-56I; doi:I0.1038/npp.20 I6.170; published online 2 I September 2016

\section{INTRODUCTION}

In our rapidly aging society, an increase of neurodegenerative disorders (eg, Alzheimer's disease, AD) is predicted. As no effective disease-modifying pharmacological intervention has been approved so far, the search for new strategies to maintain higher brain function throughout life and to prevent the progression of the 'AD-precursor', mild cognitive impairment (MCI), to full-blown dementia is of major economic and medical importance (Mangialasche et al, 2010). Cognitive training (Anguera et al, 2013) and non-invasive brain stimulation techniques, such as anodal transcranial direct

\footnotetext{
*Correspondence: Dr K Prehn, Department of Neurology and NeuroCure Clinical Research Center, Charité Universitätsmedizin Berlin, Charitéplatz I, I0117 Berlin, Germany, Tel: +49 (0)30 450560140, Fax: +49 (0)30 450560974, E-mail: kristin.prehn@charite.de or Professor A Flöel, Department of Neurology and NeuroCure Clinical Research Center, Charité Universitätsmedizin Berlin, Charitéplatz I, 10117 Berlin, Germany, Tel: +49 (0)30 450560284, Fax: +49 (0)30 4507539939, E-mail: agnes.floeel@charite.de

Received I8 June 2016; revised 28 July 20 I6; accepted 10 August 20 16; accepted article preview online 24 August 2016
}

current stimulation (atDCS; Prehn and Floel, 2015), might offer exciting opportunities for enhancing brain function. atDCS, in particular, may help to overcome reduced brain responsiveness to cognitive training in aging, as it modulates post-synaptic connections analogous to long-term potentiation (LTP; Liebetanz et al, 2002) and has been shown to improve learning and memory in young and older healthy subjects by our group and others (Miniussi et al, 2013; Coffman et al, 2014; Shin et al, 2015). It has been shown, for instance, that atDCS over the left peri-sylvian area (Wernicke's area) enhances associative verbal learning (Floel et al, 2008). Older subjects, in another study (Floel et al, 2012), forgot fewer object-location associations after atDCS over the right temporoparietal cortex compared with sham stimulation.

These effects in healthy subjects and its excellent safety profile (Fregni et al, 2015) have encouraged the use of tDCS also after stroke and in neurodegenerative disease (Floel, 2014; Prehn and Floel, 2015). For instance, atDCS has been successfully applied to enhance language training outcome in post-stroke aphasia (Floel et al, 2011; Meinzer et al, 2016). In AD patients, Ferruci et al, (2008) and Boggio et al, (2009) demonstrated in small pilot studies that episodic memory 
a Study flow

\begin{tabular}{|c|c|c|c|c|c|}
\hline $\max$. & reeks & 2-4 weet & 2-4 weeh & 2-4 wee & \\
\hline \multirow[t]{2}{*}{$\begin{array}{l}\text { V0: } \\
\text { Screening of } n=48 \\
\text { subjects }\end{array}$} & $\begin{array}{l}\text { Randomization } \\
\text { into } 4 \text { treatment } \\
\text { groups } \\
\end{array}$ & $\mathrm{V} 1^{*}$ & $\mathrm{~V}^{*}{ }^{*}$ & V3 $^{*}$ & V4* \\
\hline & & $A(n=10)$ & $B(n=10)$ & $C(n=10)$ & $D(n=10)$ \\
\hline \multirow{3}{*}{$\mathrm{n}=40 \leqq$} & $\longrightarrow 2$ & $B(n=10)$ & $D(n=10)$ & $A(n=10)$ & $C(n=10)$ \\
\hline & $\longrightarrow 3$ & $C(n=10)$ & $A(n=9)$ & $D(n=9)$ & $B(n=9)$ \\
\hline & $\rightarrow 4$ & $D(n=10)$ & $C(n=10)$ & $B(n=10)$ & $A(n=10)$ \\
\hline
\end{tabular}

$$
\begin{aligned}
& A=\text { sham }+ \text { placebo } \\
& B=\text { sham+SSRI } \\
& C=\text { atDCS+placebo } \\
& D=\text { atDCS+SSRI }
\end{aligned}
$$

b Course of an experimental visit (V1, V2, V3, V4)

Drug intake

$$
\text { SSRI vs. placebo }
$$

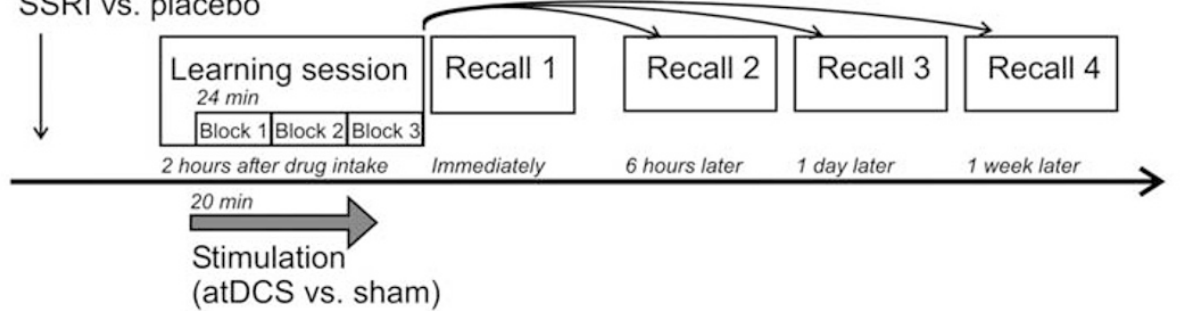

*Experimental visit comprising a learning session and immediate and delayed cued recalls (see b)

(atDCS vs. sham)

Figure I Study flow (a) and course of an experimental visit (b). (a) During a screening visit (V0), inclusion and exclusion criteria were evaluated for each participant on the basis of anamnesis, medical and neuropsychological examination, electrocardiogram, magnetic resonance imaging (MRI) scan, and blood tests. From 48 screened subjects, 40 subjects were included and randomized into four treatment groups (with 5 young and 5 older subjects each) with four different sequences of intervention conditions ( A, B, C, and D). Maximal 2 weeks after V0, the four experimental visits (VI, V2, V3, and V4) were scheduled. (b) Each experimental visit comprised a learning session during which participants performed the objects-location learning (LOCATO) task with brain stimulation (anodal transcranial direct current stimulation (atDCS) vs sham) and drug intake (selective serotonin reuptake inhibitor (SSRI) vs placebo) $2 \mathrm{~h}$ before. The learning session consisted of three learning blocks and was followed by immediate and delayed cued recall tasks (recall I, 2, 3, and 4). Between the experimental visits (VI, V2, V3, and V4) was a wash-out phase of at least 2 weeks (maximum 4 weeks).

performance can be enhanced by atDCS administration over the dorsolateral prefrontal, temporal, and temporoparietal cortex. Effect sizes, however, were only small to moderate and producing sustained improvements of training success remains challenging (Prehn and Floel, 2015).

Different approaches to reinforce and prolong the effects of atDCS have been suggested. First, repeated stimulation protocols or even daily applications over a longer intervention period seem to be promising (Reis et al, 2009; Cohen Kadosh et al, 2010; Meinzer et al, 2014). Second, the mechanisms involved in atDCS (ie, alteration of resting membrane potential and LTP-like synaptic plasticity) are also susceptible to dopaminergic (Kuo et al, 2008) and serotonergic modulation (Nitsche et al, 2009; Kuo et al, 2016). Serotonin, in particular, has an important role in learning and memory, as well as in mood regulation, depression, and anxiety (Foehring and Lorenzon, 1999). Blocking the serotonin transporter with a selective serotonin receptor inhibitor (SSRI) raises the level of serotonin available in many structures of the brain (eg, frontal cortex, striatum, hippocampus, and raphe nucleus; Invernizzi et al, 1997). Although it usually takes some time until SSRIs reduce the clinical symptoms of depression, effects on cognition (Outhred et al, 2014; Jonassen et al, 2015) and functional neural networks (Klaassens et al, 2015) have already been described after single-dose administration. A study published by Nitsche et al (2009), eg, demonstrated that atDCSinduced enhancement of motor cortex excitability (monitored via motor-evoked potentials, MEPs) can be amplified and prolonged by a single dose of $20 \mathrm{mg}$ citalopram. The extent, however, to which SSRIs also modulate the effect of atDCS on higher cognitive functions remains unclear.

In the present study, we investigated the effect of atDCS over the right temporoparietal cortex and SSRI application (single dose of $20 \mathrm{mg}$ citalopram) on memory performance. We hypothesized that a combined application of atDCS and SSRI would result in enhanced immediate recall scores compared with the application of atDCS alone in young and older subjects (primary hypothesis). In addition to enhancement of immediate recall performance, we expected to find enhanced delayed recall scores in this intervention condition (secondary hypothesis).

\section{MATERIALS AND METHODS}

\section{Study Overview}

The study employed a placebo-/sham-controlled, doubleblind within-subjects design and was conducted at Charité Universitätsmedizin Berlin. Twenty young and 20 older participants completed a standardized object-location learning task in four intervention conditions: sham+placebo, 
Table I Demographic and Neuropsychological Characteristics (mean (SD)) for Young and Older Participants

\begin{tabular}{|c|c|c|c|}
\hline & Young adults $n=20$ mean (SD) & Older adults $n=20$ mean (SD) & $p$-value \\
\hline Age (years) & $24(4)$ & $66(7)$ & $<0.001$ \\
\hline Gender (women/men) & $12 / 8$ & $11 / 9$ & $0.75^{\mathrm{a}}$ \\
\hline TAP: alertness (ms) & $238(31)$ & $280(63)$ & 0.01 \\
\hline TAP: divided attention (ms) & $716(70)$ & $884(102)$ & $<0.001$ \\
\hline TAP: nback (ms) & $473(125)$ & $528(136)$ & 0.19 \\
\hline Digit span forward (no. of digits) & $8.5(2.0)$ & $8.4(1.4)$ & 0.86 \\
\hline Digit span backward (no. of digits) & $7.6(2.4)$ & $7.0(1.6)$ & 0.36 \\
\hline Visual spatial memory span (no. of items) & $6.0(0.6)$ & $5.5(0.7)$ & 0.02 \\
\hline Rey-Osterrieth figure: copy (score) & $35.9(0.3)$ & $35.5(0.6)$ & 0.03 \\
\hline Rey-Osterrieth figure: recall (score) & $25.7(4.6)$ & $26.1(3.8)$ & 0.77 \\
\hline VLMT: recognition (no. of words) & | $4.7(0.9)$ & $14.3(1.0)$ & 0.25 \\
\hline Depression (Beck's Depression Index) & $1.7(2.0)$ & $4.6(3.5)$ & 0.002 \\
\hline WHO Quality of Life (score) & $105.6(13.7)$ & $103.8(11.5)$ & 0.64 \\
\hline Pittsburgh Sleep Quality (score) & $4.2(1.8)^{\mathrm{a}}$ & $4.9(2.5)$ & 0.36 \\
\hline
\end{tabular}

Abbreviations: TAP, test battery for attentional performance; VLMT, verbal learning and memory test.

A $p$-value $\leqslant 0.05$ indicates a significant difference between the groups calculated with a $t$-test for independent samples unless otherwise noted.

${ }^{\mathrm{a} C}$ Calculated using a $\chi^{2}$-test.

sham+SSRI, atDCS+placebo, and atDCS+SSRI. To avoid order effects, participants were randomly assigned to four groups with four different sequences of conditions over the four visits (V1, V2, V3, and V4) using a Williams design (Williams, 1949). Experimental visits were separated by a wash-out phase of at least 2 weeks (maximum 4 weeks) to avoid cumulative SSRI or atDCS effects. For an overview of the study flow, see Figure 1a.

\section{Participants}

Twenty young (18-35 years) and 20 older (50-80 years) healthy subjects participated in the study. Participants were all right-handed as assessed using the Edinburgh Handedness Inventory (Oldfield, 1971) and had no history of chronic or acute neurologic, psychiatric, or medical disease. Inclusion and exclusion criteria were first checked during a telephone screening (for a complete list, see Supplementary Material). At the screening visit (V0), eligible subjects underwent a medical interview, a neurological examination, comprehensive neuropsychological testing (Supplementary Material and Table 1), electrocardiography, magnetic resonance imaging (MRI) of the brain, and blood sampling (recruitment period: April 2014 to September 2014).

The study was registered at https://clinicaltrials.gov (NCT02092974; name of trial registry: LESO) and conducted in accordance with the Good Clinical Practice guidelines of the Food and Drug Administration and the International Conference on Harmonization. The protocol and all studyspecific materials were approved by the German Federal Institute for Drugs and Medical Devices (BfArM) and the Regional Office for Health and Social Affairs in Berlin
(LaGeSo; EudraCT: 2013-004821-10). All subjects provided written informed consent before study-specific procedures were conducted and received a small reimbursement for participation.

\section{LOCATO Task and Assessment of Primary and Secondary Outcomes}

To investigate memory processing, we used the LOCATO task, which has been developed in our research group and used in similar versions in previous studies (Floel et al, 2012; Kulzow et al, 2014). This task comprised a learning session, during which participants learned the locations of 30 buildings on a schematic map, and four sessions in which learning success was tested using cued recall. In detail, learning success was tested immediately after learning (immediate recall scores = primary outcome; see also clinicaltrials.gov, identifier: NCT02092974), at the evening of the same day (ie, at least $6 \mathrm{~h}$ later), at the next morning (1 day later), and 1 week later (delayed recall scores = secondary outcomes; Figure 1b).

The learning session consisted of three blocks with 120 trials each. During a learning trial, a street map with one building was shown for $3 \mathrm{~s}$ and subjects indicated whether the building was presented at the 'correct' or 'incorrect' location by pressing one of two buttons of a response device. Over the course of the entire learning session, each building was shown six times at one specific (ie, the 'correct') location and six times at other incorrect locations (ie, six times more frequently at the correct than incorrect locations, at which the building was shown only once). Subjects were instructed to find out the correct location, but were not informed about the underlying statistical principle, and did not receive any 
feedback. During the recall sessions, each building and the street map together with three possible locations were presented, and subjects indicated the correct position by button press. The three-alternative response format was used here in contrast to previous versions, in which free recall was used (Floel et al, 2012), to make the scoring easier and more objective.

Previous studies demonstrated long-term test-retest reliability of LOCATO (Kulzow et al, 2014), the usefulness of the LOCATO outcome measures (learning performance, immediate, and delayed recall scores) to differentiate between patients with MCI and healthy subjects (Kulzow et al, 2014), and to detect changes in memory performance induced by brain stimulation (Floel et al, 2012) and dietary supplements (Kulzow et al, 2016).

LOCATO was presented using Presentation (Neurobehavioral Systems, Albany, CA, USA). As each subject completed the task in each of the four experimental conditions, we created four parallel LOCATO versions with a different set of pictures and a different schematic street map (created by flipping the original map horizontally, vertically, and both), which were assigned to the intervention conditions in a pseudo-randomized order.

\section{atDCS Administration}

atDCS was administered through a direct current stimulator (DC-Stimulator Plus, NeuroConn GmbH, Ilmenau, Germany) and two electrodes, which were inserted into synthetic sponges soaked with saline solution and attached to the scalp using rubber bands. The stimulating electrode (to which the term 'anodal stimulation' refers, $6.5 \times 6.5 \mathrm{~cm}$ ) was centered over the right temporoparietal area (T6, according to the international 10-20 electroencephalography system). This area has been shown to be important for object-location learning in the literature (Postma et al, 2008; van Asselen et $a l, 2008$ ) and a previous atDCS study of our group (Floel et al, 2012). The second electrode (reference, $9.5 \times 9.5 \mathrm{~cm}$ ) was placed over the contralateral frontopolar cortex. The size of the reference electrode was chosen to be larger as the size of the anode to render stimulation over this cortical area functionally inefficient (Nitsche et al, 2007). During atDCS stimulation, a constant direct current of $1 \mathrm{~mA}$ was applied for $20 \mathrm{~min}$ during the learning session (in accordance with Nitsche et al, 2009, who previously showed that citalopram enhances tDCS-induced motor cortex excitability). The current was increased over $10 \mathrm{~s}$ at the beginning of the stimulation (fade in) in a ramp-like fashion and decreased over $5 \mathrm{~s}$ at the end (fade out). During sham stimulation, the procedure was essentially the same with the only difference being that the current was ramped down automatically already after $30 \mathrm{~s}$, allowing efficient blinding of participants and instructors (Gandiga et al, 2006).

\section{SSRI Administration}

Citalopram $(20 \mathrm{mg})$ or placebo drugs were administered orally in a double-blind fashion $2 \mathrm{~h}$ before the start of the learning session on all four experimental visits, allowing citalopram to induce stable plasma levels (BezchlibnykButler et al, 2000).

\section{Experimental Procedures}

For each experimental visit, participants arrived in the morning at the laboratory (between 0800 and 1200 hours). After receiving SSRI/placebo medication, subjects were instructed and completed a short practice session. Then, the electrodes were placed on the participant's head. Two hours after SSRI/placebo intake, the learning session was started. Stimulation was started together with the start of the first learning block. After the learning session, the electrodes were removed. Subjects then performed the immediate cued recall task and were scheduled for delayed recalls. To assess whether intervention conditions caused changes in mood, participants completed the Visual Analogue Mood Scales (VAMS; Stern et al, 1997) before and after each experimental visit. After the last visit (V4), side effects for stimulation and medication were recorded using two scales. The scale for stimulation-induced side effects was adopted from Poreisz et al (2007) and contained questions regarding the presence of specific symptoms such as tiredness, headache, difficulties in concentrating, visual perceptual changes, and discomforting sensation (ie, itching or burning) under the electrodes. The possibility to name further side effects in an openresponse format was also given. The scale for medicationinduced side effects asked whether there were any side effects or changes in feelings and experiences using an openresponse format (eg, 'Did you observe any changes in feelings after the drug intake?') which allowed for the assessment of symptoms without potential priming effects of a pre-constructed symptom list. Subjects were also asked to indicate at which visit each side effect occurred. At the end of each scale, subjects indicated whether they were able to distinguish between stimulation and medication conditions. If so, they were asked to describe how they knew (eg, at which of the four visits they thought they had received atDCS and/or citalopram). Although subjects reported side effects for stimulation and medication separately, symptoms cannot be separated unambiguously, as stimulation and medication were combined and sham/placebo application could also have induced side effects.

\section{Data Analysis and Statistics}

The immediate recall scores (numbers of correctly identified locations in \% correct) were analyzed with a linear mixed model (random intercept model; Verbeke and Molenberghs, 2000) computed in SPSS 22.0 (PASW, SPSS; IBM, Armonk, NY). In this model, repeated measurements (157 data points in total; $n=20$ young and $n=20$ older subjects) assessed during the four experimental visits were entered as level one units nested in the different individuals, which were entered as level two units. The mixed model included the factor 'intervention condition' (with four levels: sham+placebo, sham+SSRI, atDCS+placebo, and atDCS+SSRI), the factor 'group' (young $v s$ older subjects), and a centered factor 'visit', which adjusted for a linear test-retest effect over the four experimental visits. To account for individual differences in learning ability, we used individual numbers of correct responses (hits plus correct rejections) in the third learning block as a centered covariate (in the following referred to as 'learning performance'). 
Pair-wise model-based post hoc tests were computed to compare the effects of the different intervention conditions. If the main effect of condition was not significant (which was the case for all secondary outcome measures), post hoc tests were only computed in an exploratory way. Effect sizes (Hedges' $g$ ) for differences between conditions were calculated using post hoc marginal means and SE of these means. To infer the amount of variance explained by our model, we computed marginal and conditional $R^{2}$ measures as proposed by Nakagawa and Schielzeth (2013) and Johnson (2014). Marginal $R^{2}$ reflects the variance that can be attributed to the fixed effects of the model, whereas conditional $R^{2}$ includes additional variance that is explained by random effects. To also assess the overall SSRI and atDCS effects, we computed an additional linear mixed model with the factors 'stimulation condition' (atDCS $v s$ sham), 'medication condition' (SSRI $v s$ placebo), 'group' (young vs older subjects), 'visit', and the covariate 'learning performance'.

Delayed recall scores (secondary outcomes) were also analyzed using linear mixed models with the factors 'intervention condition,' 'group,' 'visit,' and 'learning performance' and a linear mixed model with the factors 'stimulation condition' (atDCS vs sham), 'medication condition' (SSRI vs placebo), 'group' (young vs older subjects), 'visit', and the covariate 'learning performance'. We also determined the effect of the factors 'intervention', 'stimulation', 'medication condition', 'group', and 'visit' on learning performance and changes in positive and negative mood with linear mixed models (also always including 157 data points in total). No adjustments were made to correct for multiple comparisons. The two-sided level of significance for all analyses was set at $\alpha=0.05$.

\section{RESULTS}

\section{General Effects of atDCS and SSRI Administration}

All subjects tolerated atDCS and citalopram medication well. No serious adverse events were reported. Due to brain stimulation, 17 young and 9 older subjects indicated that they had experienced tiredness $(12 \times)$, discomforting sensations such as itching and burning under the electrodes $(11 \times)$, difficulties in concentrating $(10 \times)$, and mild headache $(2 \times)$. Due to medication, 8 young and 3 older subjects reported nausea $(7 \times)$, dizziness $(6 \times)$, difficulties in concentrating $(2 \times)$, sweating $(1 \times)$, mild headache $(1 \times)$, a feeling of higher activation $(1 \times)$, and tiredness $(1 \times)$.

At the end of the study, 12 subjects reported that they were able to distinguish atDCS from sham stimulation, but only 3 of them were correct (ie, indicated the correct sequence of stimulation conditions or reported that they recognized atDCS because of specific side effects that were reported for the correct sessions). Eight subjects reported that they were able to distinguish citalopram from placebo and all of them were correct. One subject among those could identify both interventions correctly.

\section{atDCS and SSRI Administration Effects on Immediate Recall (Primary Hypothesis)}

The mixed model revealed a significant main effect of intervention condition $(F(3,106)=2.9, \quad p=0.04)$ on

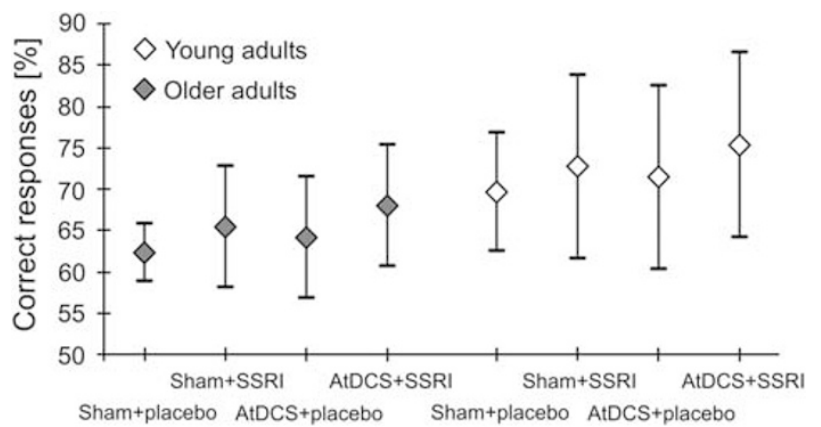

Figure 2 Immediate recall scores (primary outcome) for the four intervention conditions. Displayed is the mean score (in \% correct responses, 95\% Cl) for each condition in young $(n=20)$ and older participants $(n=20)$ based on the calculated mixed linear model (ie, values adjusted for individual learning performance measured by learning success at the end of the last learning block) and test-retest effect over the four experimental visits comprising 157 data points in total.

immediate recall scores (Figure 2). Post hoc comparisons of conditions revealed that subjects showed improved recall performance in the atDCS+SSRI condition compared with atDCS+placebo (Tables $2 \mathrm{~A}$ and $3 \mathrm{~A}$ ). In detail, the SSRI tatDCS condition increased immediate recall scores on average by $\sim 4 \%$ compared with atDCS+placebo. tDCS+SSRI compared with sham+placebo even improved recall on average by $\sim 6 \%$. We did not find a significant difference between the conditions tDCS+placebo and sham+placebo and between sham+SSRI and sham+placebo; ie, no significant effects of SSRI and tDCS alone could be detected. The comparison atDCS+SSRI $>$ sham + SSRI did not reveal a significant difference.

In addition, we found significant effects of the group $(F(1,33)=14.3, p=0.001)$ and visit $(F(1,129)=5.0, p=0.03)$. The effect of the group indicates that younger subjects recalled locations better than older ones (mean difference between the groups: $\sim 7 \%$ ). The effect of visit shows that all subjects improved in recall performance over the four visits (mean increase in recall scores per visit: $\sim 1.5 \%$ ). We additionally tested for an interaction between factors group and intervention condition. There was no significant interaction $(F(3,103)=0.1, p=0.97)$, indicating that the effect of condition did not differ between the groups. The model also revealed a significant effect of learning performance $(F(1,49)=240.8, p<0.001)$, showing that successful recall of object-location associations needs successful learning of these associations before. Marginal and conditional $R^{2}$ $\left(R^{2} \mathrm{~m}=0.79 ; R^{2} \mathrm{c}=0.81\right)$ of the mixed linear model indicated that the model explained the data in a sufficient way.

Calculating the effect of stimulation and medication, we found a significant effect of SSRI on immediate recall scores $(F(1,107)=6.3, p=0.013)$, indicating higher scores in the SSRI compared with the placebo conditions, but no significant effect of atDCS $(F(1,107)=2.5, p=0.12)$.

\section{atDCS and SSRI Administration Effects on Delayed Recall (Secondary Hypothesis)}

The mixed linear models on delayed recall scores (secondary outcomes) revealed no significant main effect of intervention condition, stimulation, and medication condition. There was 
Table 2 Mean (SD) Immediate Recall Scores (A, Primary Outcome), Delayed Recall Scores (B, Secondary Outcomes), Learning Performance (C), and Changes in Mood (D) for Young $(n=20)$ and Older Subjects $(n=20)$ in the Four Intervention Conditions (sham + placebo, sham+SSRI, atDCS+placebo, and atDCS+SSRI)

\begin{tabular}{|c|c|c|c|c|c|c|c|c|}
\hline & \multicolumn{2}{|c|}{ sham+placebo } & \multicolumn{2}{|c|}{ sham+SSRI } & \multicolumn{2}{|c|}{ atDCS+placebo } & \multicolumn{2}{|c|}{ atDCS+SSRI } \\
\hline & $\begin{array}{l}\text { Young } \\
(n=20)\end{array}$ & $\begin{array}{c}\text { Older } \\
(n=19)\end{array}$ & $\begin{array}{l}\text { Young } \\
(n=20)\end{array}$ & $\begin{array}{c}\text { Older } \\
(n=20)\end{array}$ & $\begin{array}{c}\text { Young } \\
(n=20)\end{array}$ & $\begin{array}{c}\text { Older } \\
(n=20)\end{array}$ & $\begin{array}{l}\text { Young } \\
(n=20)\end{array}$ & $\begin{array}{c}\text { Older } \\
(n=19)\end{array}$ \\
\hline (A) Immediate recall scores & $77.67(21.52)$ & $57.19(14.96)$ & $82.67(11.83)$ & $57.54(17.74)$ & $80.33(20.34)$ & $57.00(15.67)$ & $80.00(18.19)$ & $60.00(15.28)$ \\
\hline \multicolumn{9}{|l|}{ (B) Delayed recall scores } \\
\hline 6 h later & $78.33(20.73)$ & $54.91(11.99)$ & $80.67(14.29)$ & $54.04(14.55)$ & $80.00(21.41)$ & $54.56(16.93)$ & $77.83(14.48)$ & $54.04(16.98)$ \\
\hline I day later & $74.67(21.83)$ & $52.11(13.93)$ & $80.00(15.10)$ & $53.15(13.98)$ & $79.33(20.62)$ & $51.05(16.56)$ & $76.50(17.95)$ & $51.05(16.74)$ \\
\hline I week later & $69.00(23.42)$ & $48.60(9.90)$ & $71.17(17.45)$ & $46.14(12.87)$ & $69.33(22.62)$ & $48.95(15.64)$ & $69.17(17.20)$ & $49.47(15.68)$ \\
\hline (C) Learning performance & $81.88(14.17)$ & $70.57(7.21)$ & $82.83(11.15)$ & $68.82(9.39)$ & $82.08(13.42)$ & $69.42(7.88)$ & $79.17(11.96)$ & $68.07(11.16)$ \\
\hline \multicolumn{9}{|l|}{ (D) Changes in mood } \\
\hline VAMS positive & $-1.35(18.74)^{a}$ & $0.96(28.35)$ & $9.00(19.43)$ & | 1.58 (26.19) & $5.67(21.85)$ & $6.67(20.33)$ & $7.58(11.95)$ & $3.86(14.07)$ \\
\hline VAMS negative & $0.92(5.46)^{b}$ & $-2.43(5.05)$ & $0.25(10.49)$ & $-1.79(9.76)$ & $2.44(6.23)$ & $-1.28(7.44)$ & $4.86(6.69)$ & $-2.49(7.87)$ \\
\hline
\end{tabular}

${ }^{a}$ Negative values indicate an increase in positive/negative mood in the respective condition during the experimental session (pre-post difference).

${ }^{b}$ Positive values indicate a decrease in positive/negative mood in the respective condition during the experimental session (pre-post difference).

a small but not significant overall SSRI effect at the next morning $(F(1,108)=3.5, p=0.06)$. Exploratory post hoc tests also showed some improvement in the conditions atDCS +SSRI and sham+SSRI both compared with sham+placebo (Tables 2B and 3B).

All models testing for effects on delayed recall showed significant group effects (all $p s<0.004)$ and significant effects of learning performance (all $p s<0.001$ ), similarly as for immediate recall scores. However, we did not detect significant changes in delayed recalls over the four visits (all $p s>0.19$ ).

\section{Further Effects of atDCS and SSRI Administration on Learning Performance and Mood}

The linear mixed models on learning performance revealed no significant effects of intervention condition $(F(3,114)=2.3, \quad p=0.08)$, and no significant effects of stimulation $(F(1,115)=2.9, \quad p=0.094)$ and medication $(F(1,115)=2.9, p=0.090)$. Exploratory post hoc comparisons, however, demonstrated reduced learning performance during atDCS+SSRI compared with sham+placebo (mean difference: $\sim 3 \%$; Tables $2 \mathrm{C}$ and $3 \mathrm{C}$ ). We also found some reduction in learning performance in condition atDCS+SSRI compared with atDCS+placebo and sham+SSRI (for both comparisons: mean difference: $\sim 2 \%$ ). Results indicate that learning was affected when the SSRI was administered together with atDCS. atDCS application per se did not significantly influence learning performance.

We also found a significant effect of the group $(F(1,151)=53.0, \quad p<0.001) \quad$ and visit $\quad(F(1,151)=14.8$, $p<0.001)$, indicating better learning performance in young subjects (difference between the groups: $\sim 12 \%$ ) and improvement in learning performance over the four visits (mean increase in learning performance per visit: $\sim 2 \%$ ). Finally, learning performance (averaged across the four visits) correlated with individual visuo-spatial abilities assessed with the Rey-Osterrieth Complex Figure Task (copying: Pearson's $r=0.38, p=0.02$; recall: Pearson's $r=0.39, p=0.02)$. These associations provide evidence for the external validity of the LOCATO task, and reflect the usefulness of the task to assess clinically relevant visuospatial abilities.

We neither found a significant effect of intervention condition, nor an effect of the group, nor an effect of visit on positive and negative mood (all $p s>0.16$ ). Effects of stimulation and medication were also not found. Exploratory post hoc comparisons, however, showed a significant decrease in positive mood when comparing condition sham+SSRI with sham+placebo (Tables 2D and 3D). Positive mood was also lower in conditions atDCS+SSRI and atDCS+placebo compared with sham+placebo, although this was not statistically significant. Finally, we found a significant effect of the group $(F(1,38)=7.3, p=0.01)$, indicating that older subjects reported a higher level of tiredness and exhaustion after the experimental visits than younger subjects.

\section{DISCUSSION}

The present study yielded two main results: First, we found an effect of medication but no significant effect of stimulation on immediate recall scores. However, both young and older subjects benefited most from the combination of interventions (ie, from condition atDCS+SSRI). Second, we found no effects (other than a trend for the comparison atDCS+SSRI $>$ sham + placebo) on delayed recalls. In other words, our data provide first evidence that atDCS improves immediate memory performance if serotonergic neurotransmission is enhanced simultaneously, but these effects do not persist over time.

\section{Effects of atDCS and Serotonergic Enhancement on Immediate Memory Performance}

The result of an effect of atDCS+SSRI on immediate recall (comparisons: atDCS+SSRI > atDCS+placebo and atDCS+SSRI > sham+placebo) in both young and older subjects is in line with Nitsche et al (2009), who also found that a single dose of citalopram enhanced tDCS-induced neuroplasticity as indicated by larger MEP amplitudes. 
Table 3 Overall atDCS and SSRI Effects (in bold) and Post hoc Comparisons for Effects of Intervention Conditions Based on the Linear Mixed Models (Mean Difference Scores in \% Correct Responses (SE), p-values, g) for Immediate Recall Scores (A, Primary Outcome), Delayed Recall Scores (B, Secondary Outcomes), Learning Performance (C), and Changes in Mood (D)

\begin{tabular}{|c|c|c|c|}
\hline & Mean difference (SE) & $p$-value & Effect size Hedges' g \\
\hline \multicolumn{4}{|l|}{ (A) Immediate recall scores } \\
\hline atDCS $>$ sham & $2.19(1.40)$ & 0.120 & 0.24 \\
\hline SSRI > placebo & $3.51(1.40)$ & 0.013 & 0.39 \\
\hline atDCS+SSRI > atDCS+placebo & $3.92(1.98)$ & 0.050 & 0.31 \\
\hline atDCS+SSRI > sham+SSRI & $2.60(1.99)$ & 0.194 & 0.20 \\
\hline atDCS+SSRI > sham+placebo & $5.70(1.99)$ & 0.005 & 0.45 \\
\hline atDCS+ placebo > sham+SSRI & $-1.32(1.97)$ & 0.506 & -0.11 \\
\hline atDCS+placebo > sham+placebo & $1.79(1.97)$ & 0.367 & 0.14 \\
\hline sham+SSRI > sham+placebo & $3.10(1.98)$ & 0.121 & 0.25 \\
\hline \multicolumn{4}{|l|}{ (B) Delayed recall scores } \\
\hline \multicolumn{4}{|l|}{6 h later } \\
\hline atDCS $>$ sham & $1.02(1.33)$ & 0.446 & 0.12 \\
\hline SSRI > placebo & $1.25(1.33)$ & $0.35 I$ & 0.14 \\
\hline atDCS+SSRI > atDCS+placebo & $1.36(1.89)$ & 0.474 & 0.11 \\
\hline atDCS+SSRI > sham+SSRI & $1.13(1.89)$ & 0.552 & 0.09 \\
\hline atDCS+SSRI > sham+placebo & $2.27(1.90)$ & 0.234 & 0.19 \\
\hline atDCS+placebo > sham+SSRI & $-0.22(1.89)$ & 0.904 & -0.02 \\
\hline atDCS+placebo > sham+placebo & $0.91(1.89)$ & 0.630 & 0.08 \\
\hline sham+SSRI > sham+placebo & $1.14(1.89)$ & 0.547 & 0.09 \\
\hline \multicolumn{4}{|l|}{ I day later } \\
\hline atDCS > sham & I.II (1.38) & 0.423 & 0.12 \\
\hline SSRI > placebo & $2.58(1.38)$ & 0.063 & 0.29 \\
\hline atDCS+SSRI > atDCS+placebo & $1.49(1.94)$ & 0.445 & 0.12 \\
\hline atDCS+SSRI > sham+SSRI & $-0.003(1.96)$ & 0.999 & -0.0002 \\
\hline atDCS+SSRI > sham+placebo & $3.68(1.95)$ & 0.061 & 0.29 \\
\hline atDCS+placebo > sham+SSRI & $-1.49(1.95)$ & 0.446 & -0.12 \\
\hline atDCS+placebo > sham+placebo & $2.19(1.94)$ & 0.260 & 0.18 \\
\hline sham+SSRI > sham+placebo & $3.69(1.95)$ & 0.061 & 0.30 \\
\hline \multicolumn{4}{|l|}{ I week later } \\
\hline atDCS > sham & $1.58(1.55)$ & 0.312 & 0.16 \\
\hline SSRI > placebo & $1.25(1.55)$ & 0.423 & 0.13 \\
\hline atDCS+SSRI > atDCS+placebo & $2.29(2.21)$ & 0.302 & 0.16 \\
\hline atDCS+SSRI > sham+SSRI & $2.62(2.21)$ & 0.238 & 0.19 \\
\hline atDCS+SSRI> sham+placebo & $2.85(2.22)$ & 0.201 & 0.20 \\
\hline atDCS+placebo > sham+SSRI & $0.33(2.19)$ & 0.882 & 0.02 \\
\hline atDCS+placebo > sham+placebo & $0.56(2.19)$ & 0.801 & 0.04 \\
\hline sham+SSRI>sham+placebo & $0.23(2.19)$ & 0.917 & 0.02 \\
\hline \multicolumn{4}{|l|}{ (C) Learning performance } \\
\hline atDCS $>$ sham & $-1.33(0.79)$ & 0.094 & 0.26 \\
\hline SSRI $>$ placebo & $-1.35(0.79)$ & 0.090 & 0.26 \\
\hline atDCS+SSRI > atDCS+placebo & $-2.18(1.11)$ & 0.052 & -0.31 \\
\hline atDCS+SSRI > sham+SSRI & $-2.17(1.12)$ & 0.054 & -0.30 \\
\hline atDCS+SSRI> sham+placebo & $-2.68(1.12)$ & 0.018 & -0.37 \\
\hline atDCS+placebo > sham+SSRI & $0.01(1.11)$ & 0.99 & 0.001 \\
\hline atDCS+placebo > sham+placebo & $-0.50(1.11)$ & 0.656 & -0.07 \\
\hline sham+SSRI > sham+placebo & $-0.51(1.12)$ & 0.648 & -0.07 \\
\hline
\end{tabular}




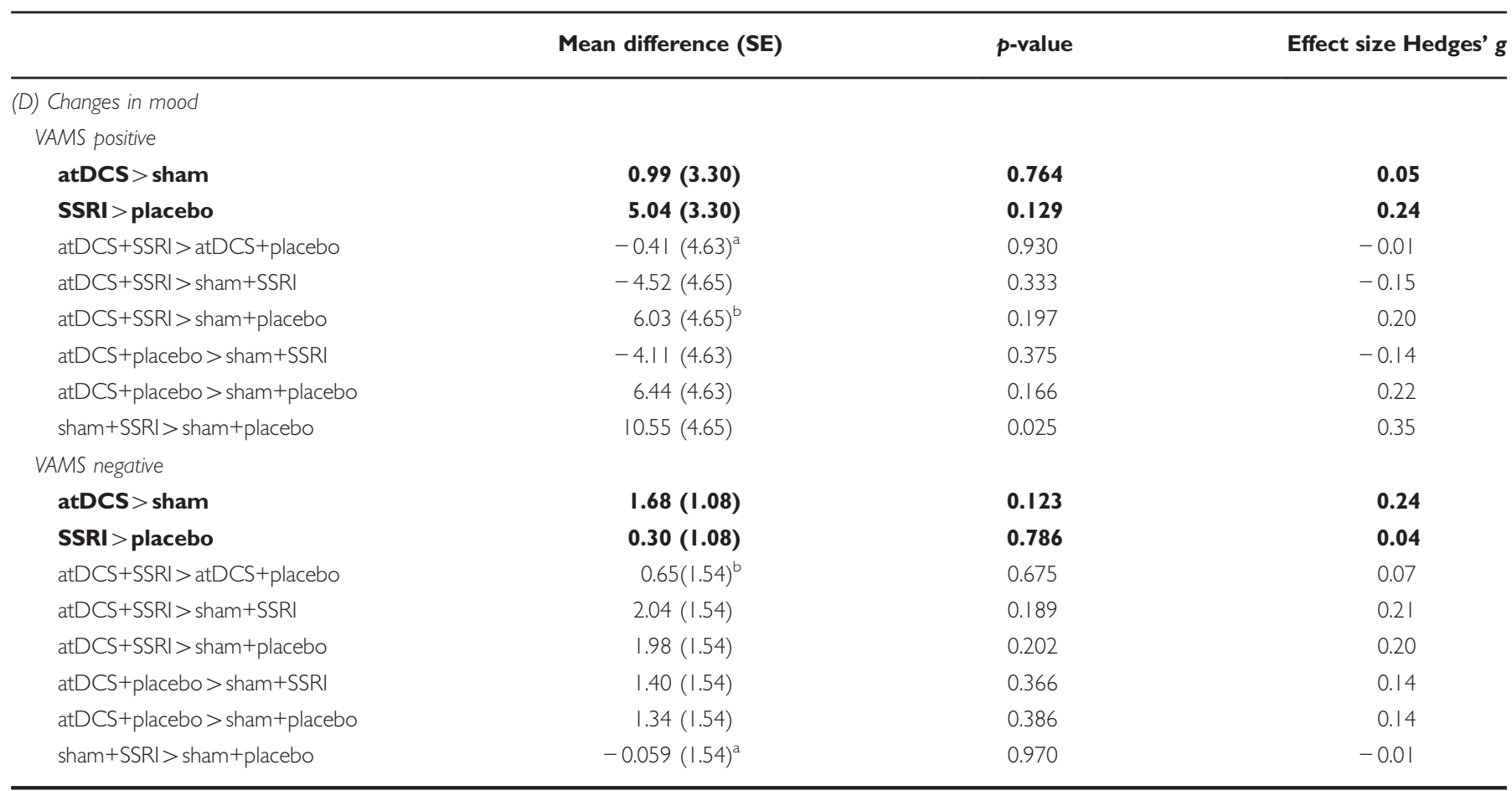

All models included $(n=20)$ young and $(n=20)$ older subjects with 157 data points in total.

a Negative difference values indicate an increase in positive/negative mood (ie, in the atDCS+SSRI compared with the atDCS+placebo condition).

${ }^{b}$ Positive difference values indicate a decrease in positive/negative mood (ie, in the atDCS+SSRI compared with the sham+placebo condition).

The serotonergic system has a modulatory role in a vast number of physiological and behavioral functions and has been, particularly, implicated in motor learning (Jacobs and Fornal, 1997). Studies in animals and slice preparations demonstrated that serotonin activates a variety of protein kinases pathways within afferent sensory neurons, which increase their excitability and facilitate synaptic transmission to motor neurons (Barbas et al, 2003). Clinically, it has been demonstrated that early administration of SSRIs (in combination with physiotherapy) accelerates recovery in patients with post-stroke motor deficits (Chollet et al, 2011; Siepmann et al, 2015). A number of studies show that antidepressant therapy with SSRIs does not only improve mood but also cognitive functions and memory performance (Doraiswamy et al, 2003; Savaskan et al, 2008). However, other studies could not confirm these results. With regard to late-life depression, in which cognitive deficits are highly prevalent (Butters et al, 2004), Butters et al (2000) could demonstrate some improvement after antidepressant medication. However, subjects did not improve beyond practice effects, and did not reach normal levels of performance. Nebes et al (2003) also found that impairment persists even after patients achieve remission. These clinical studies are in line with our results. Although we found a significant overall effect of citalopram on immediate recall scores, performance was not enhanced by SSRI alone (in the sham+SSRI condition compared with sham+placebo).

We also did not find a significant effect of atDCS alone on immediate recall (comparisons: atDCS $>$ sham and atDCS + placebo $>$ sham+placebo). This negative result adds to the growing literature of heterogeneous effectiveness of tDCS on cognitive parameters, possibly due to small but potentially crucial differences in task characteristics, stimulation sites, stimulation timing, and study populations (Horvath et al, 2015a; 2015b). Strikingly, both young and older subjects, however, benefited most from the combination of interventions (ie, from condition atDCS+SSRI compared with sham + placebo).

The atDCS+SSRI condition affected immediate recall but not learning performance. Learning performance was even lower in the atDCS+SSRI condition compared with atDCS +placebo, sham+SSRI, and sham+placebo, indicating that participants may have been distracted by a combination of the mild side effects caused by either intervention. This notion is supported by the decrease in positive mood in the conditions atDCS+SSRI, atDCS+placebo, and sham+SSRI compared with sham+placebo, which was most evident in the sham+SSRI condition. This result showing an effect on memory but not on learning performance, moreover, suggests better consolidation (which did not manifest during learning however) and decelerated decay of learned information (Reis et al, 2009; Floel et al, 2012). Using functional MRI, it has been shown that consolidation of visuo-spatial information is associated with increased activation in the right hippocampus and the temporoparietal cortex (Sommer et al, 2005; Takahashi et al, 2008).

Although effect sizes in this study were small to moderate (Hedges' $g$ between 0.3 and 0.45 ), we would like to point out that significant improvements already evolved after administration of a single dose of $20 \mathrm{mg}$ citalopram in cognitively non-impaired subjects. Effects may translate into larger effects after longer application (Kuo et al, 2016) and in 
patients already suffering from memory decline. Ecological importance of our results is underscored by the finding that performance of older subjects in condition atDCS+SSRI improved almost to the level of young participants without any intervention (Figure 2). This finding confirms the results of previous studies in older subjects and patients with MCI showing that atDCS can temporarily reverse age-associated cognitive decline (Meinzer et al, 2013; 2014).

\section{No Effects of atDCS and Serotonergic Enhancement on Delayed Memory Performance}

In contrast to Nitsche et al (2009), who demonstrated that MEP amplitude enhancement under citalopram was still present $4-5 \mathrm{~h}$ after atDCS application, we did not find increased delayed recall scores when comparing atDCS+SSRI $v s$ atDCS+placebo at any of the three later time points (ie, $6 \mathrm{~h}, 1$ day, and 1 week later).

As the after-effects of tDCS on cortical excitability involve NMDA receptor-dependent processes (Nitsche et al, 2004), pharmacological interventions involving NMDA agonists might also be suited to prolong the effect of tDCS-induced plasticity on cognitive outcome measures. Specifically, Nitsche et al (2004) demonstrated that D-cycloserine (which is a partial NMDA agonist) can be used to consolidate neuronal excitability enhancements induced by atDCS. Moreover, Kuo et al (2016) showed that chronic serotonergic enhancement after daily administration of citalopram for 35 days $(20 \mathrm{mg} /$ day $)$ in contrast to single-dose application resulted in increased LTP-like glutamatergic plasticity, which increased and prolonged atDCS-induced motor cortex excitability enhancement for over $24 \mathrm{~h}$.

The lack of an effect of atDCS alone on delayed memory performance (atDCS+placebo $>$ sham+placebo) seems also to be in contrast to our previous study (Floel et al, 2012). Here, differences in the paradigm and, particularly, in the response format (three alternative forced choice $v s$ free recall) may account for differential findings.

\section{Limitations}

First, we cannot answer the question whether stimulation of other brain regions than the right temporoparietal cortex would have resulted in similar effects. Using additional stimulation regions (eg, dorsolateral prefrontal or left temporoparietal cortex; Boggio et al, 2009) would have improved the specificity of the results and should be considered in future studies. The use of a 'polarity control' (ie, cathodal tDCS) might also provide more comprehensive information about serotonergic modulation of tDCS effects.

Second, although we used four parallel LOCATO versions, we observed that subjects improved over the course of the four visits. To rule out an influence of condition order, subjects were randomly assigned to different intervention condition sequences and adjusted for a linear test-retest effect in the linear mixed models. In future studies, a more comprehensive training/familiarization with the task before starting the intervention may dilute practice effects and provide a more reliable measure of change.

Third, as reported in the results, a number of participants indicated that they could distinguish citalopram from placebo and/or atDCS from sham application. As these subjects were not invited to guess and could also justify how they knew in a credible manner (eg, by indicating the four visits in which they received atDCS and/or citalopram correctly), we concluded that blinding was not successful in these subjects. To ensure that effects found were not influenced by the unblinding, we computed an additional linear mixed model on immediate recall scores only for those subjects who reported that they could not distinguish stimulation and/or medication conditions. This analysis also revealed a significant effect of intervention condition ( $F$ $(3,79.8)=3.2, p=0.029, n=30)$.

\section{Conclusion and Outlook}

The present study demonstrates for the first time in humans that a combined application of atDCS and citalopram enhances immediate recall performance in a visuo-spatial learning task (comparisons: atDCS+SSRI $>$ atDCS+placebo and atDCS+SSRI > sham+placebo) in young and older subjects. Delayed recall was not significantly affected.

As there is a need for developing new intervention strategies for dementia and its precursors, the potential of combined application of atDCS with SSRI should now be probed in AD and MCI patients. Further studies will also have to assess whether long-term serotonergic enhancement and repeated brain stimulation leads to sustained memory improvements (Kuo et al, 2016). Long-term application of citalopram and tDCS should also reduce mild side effects, discomfort, and distraction reported in our study that might have impaired learning. After providing first-time evidence for an effect of the atDCS+SSRI application in the cognitive domain, we now suggest using this intervention in combination with (long-term) cognitive training programs.

Although the findings of the present study need to be replicated and evaluated in larger interventional trials, we believe that combined application of SSRI and tDCS might be a promising therapeutic approach to enhance memory performance in patients with cognitive decline.

\section{FUNDING AND DISCLOSURE}

This clinical trial was supported by grants from the Deutsche Forschungsgemeinschaft (Fl 379-10/1, Fl 379-11/1, and DFGExc 257), the Bundesministerium für Bildung und Forschung (FKZ 01EO0801, 01GQ1424A, and 01GQ1420B), and the Alzheimer Forschung Initiative e.V. (AVD 115110). The authors declare no conflict of interest.

\section{ACKNOWLEDGMENTS}

We would like to thank Nadine Külzow for help with programming the LOCATO task, Regina Schlieder for administrative support, and two anonymous reviewers for their helpful comments.

\section{REFERENCES}

Anguera JA, Boccanfuso J, Rintoul JL, Al-Hashimi O, Faraji F, Janowich J et al (2013). Video game training enhances cognitive control in older adults. Nature 501: 97-101.

.


Barbas D, DesGroseillers L, Castellucci VF, Carew TJ, Marinesco S (2003). Multiple serotonergic mechanisms contributing to sensitization in aplysia: evidence of diverse serotonin receptor subtypes. Learn Mem 10: 373-386.

Bezchlibnyk-Butler K, Aleksic I, Kennedy SH (2000). Citalopram-a review of pharmacological and clinical effects. J Psychiatry Neurosci 25: 241-254.

Boggio PS, Khoury LP, Martins DC, Martins OE, de Macedo EC, Fregni F (2009). Temporal cortex direct current stimulation enhances performance on a visual recognition memory task in Alzheimer disease. J Neurol Neurosurg Psychiatry 80: 444-447.

Butters MA, Becker JT, Nebes RD, Zmuda MD, Mulsant BH, Pollock BG et al (2000). Changes in cognitive functioning following treatment of late-life depression. Am J Psychiatry 157: 1949-1954.

Butters MA, Whyte EM, Nebes RD, Begley AE, Dew MA, Mulsant BH et al (2004). The nature and determinants of neuropsychological functioning in late-life depression. Arch Gen Psychiatry 61: 587-595.

Chollet F, Tardy J, Albucher JF, Thalamas C, Berard E, Lamy C et al (2011). Fluoxetine for motor recovery after acute ischaemic stroke (FLAME): a randomised placebo-controlled trial. Lancet Neurol 10: $123-130$.

Coffman BA, Clark VP, Parasuraman R (2014). Battery powered thought: enhancement of attention, learning, and memory in healthy adults using transcranial direct current stimulation. Neuroimage 85: 895-908.

Cohen Kadosh R, Soskic S, Iuculano T, Kanai R, Walsh V (2010). Modulating neuronal activity produces specific and long-lasting changes in numerical competence. Curr Biol 20: 2016-2020.

Doraiswamy PM, Krishnan KRR, Oxman T, Jenkyn LR, Coffey DJ, Burt $\mathrm{T}$ et al (2003). Does antidepressant therapy improve cognition in elderly depressed patients? J Gerontol A Biol Sci Med Sci 58: 1137-1144.

Ferrucci R, Mameli F, Guidi I, Mrakic-Sposta S, Vergari M, Marceglia S et al (2008). Transcranial direct current stimulation improves recognition memory in Alzheimer disease. Neurology 71: 493-498.

Floel A (2014). tDCS-enhanced motor and cognitive function in neurological diseases. Neuroimage 85: 934-947.

Floel A, Meinzer M, Kirstein R, Nijhof S, Deppe M, Knecht S et al (2011). Short-term anomia training and electrical brain stimulation. Stroke 42: 2065-2067.

Floel A, Rosser N, Michka O, Knecht S, Breitenstein C (2008). Noninvasive brain stimulation improves language learning. J Cogn Neurosci 20: 1415-1422.

Floel A, Suttorp W, Kohl O, Kurten J, Lohmann H, Breitenstein C et al (2012). Non-invasive brain stimulation improves object-location learning in the elderly. Neurobiol Aging 33: $1682-1689$.

Foehring RC, Lorenzon NM (1999). Neuromodulation, development and synaptic plasticity. Can J Exp Psychol 53: 45-61.

Fregni F, Nitsche MA, Loo CK, Brunoni AR, Marangolo P, Leite J et al (2015). Regulatory considerations for the clinical and research use of transcranial direct current stimulation (tDCS): review and recommendations from an expert panel. Clin Res Regul Aff 32: 22-35.

Gandiga PC, Hummel FC, Cohen LG (2006). Transcranial DC stimulation (tDCS): a tool for double-blind sham-controlled clinical studies in brain stimulation. Clin Neurophysiol 117: 845-850.

Horvath JC, Forte JD, Carter O (2015a). Evidence that transcranial direct current stimulation (tDCS) generates little-to-no reliable neurophysiologic effect beyond MEP amplitude modulation in healthy human subjects: a systematic review. Neuropsychologia 66: 213-236.

Horvath JC, Forte JD, Carter O (2015b). Quantitative review finds no evidence of cognitive effects in healthy populations from single-session transcranial direct current stimulation (tDCS). Brain Stimul 8: 535-550.

Invernizzi R, Velasco C, Bramante M, Longo A, Samanin R (1997). Effect of 5-HT1A receptor antagonists on citalopram-induced increase in extracellular serotonin in the frontal cortex, striatum and dorsal hippocampus. Neuropharmacology 36: 467-473.

Jacobs BL, Fornal CA (1997). Serotonin and motor activity. Curr Opin Neurobiol 7: 820-825.

Johnson PC (2014). Extension of Nakagawa \& Schielzeth's R2 GLMM to random slopes models. Methods Ecol Evol 5: 944-946.

Jonassen R, Chelnokova O, Harmer C, Leknes S, Landro NI (2015). A single dose of antidepressant alters eye-gaze patterns across face stimuli in healthy women. Psychopharmacology 232: 953-958.

Klaassens BL, van Gorsel HC, Khalili-Mahani N, van der Grond J, Wyman BT, Whitcher B et al (2015). Single-dose serotonergic stimulation shows widespread effects on functional brain connectivity. Neuroimage 122: 440-450.

Kulzow N, Kerti L, Witte VA, Kopp U, Breitenstein C, Floel A (2014). An object location memory paradigm for older adults with and without mild cognitive impairment. J Neurosci Methods 237: 16-25.

Kulzow N, Witte AV, Kerti L, Grittner U, Schuchardt JP, Hahn A et al (2016). Impact of omega-3 fatty acid supplementation on memory functions in healthy older adults. J Alzheimers Dis 51: 713-725.

Kuo HI, Paulus W, Batsikadze G, Jamil A, Kuo MF, Nitsche MA (2016). Chronic enhancement of serotonin facilitates excitatory transcranial direct current stimulation-induced neuroplasticity. Neuropsychopharmacology 41: 1223-1230.

Kuo MF, Paulus W, Nitsche MA (2008). Boosting focally-induced brain plasticity by dopamine. Cereb Cortex 18: 648-651.

Liebetanz D, Nitsche MA, Tergau F, Paulus W (2002). Pharmacological approach to the mechanisms of transcranial DCstimulation-induced after-effects of human motor cortex excitability. Brain 125: 2238-2247.

Mangialasche F, Solomon A, Winblad B, Mecocci P, Kivipelto M (2010). Alzheimer's disease: clinical trials and drug development. Lancet Neurol 9: 702-716.

Meinzer M, Darkow R, Lindenberg R, Floel A (2016). Electrical stimulation of the motor cortex enhances treatment outcome in post-stroke aphasia. Brain 139: 1152-1163.

Meinzer M, Jahnigen S, Copland DA, Darkow R, Grittner U, Avirame $\mathrm{K}$ et al (2014). Transcranial direct current stimulation over multiple days improves learning and maintenance of a novel vocabulary. Cortex 50: 137-147.

Meinzer M, Lindenberg R, Antonenko D, Flaisch T, Floel A (2013). Anodal transcranial direct current stimulation temporarily reverses age-associated cognitive decline and functional brain activity changes. J Neurosci 33: 12470-12478.

Miniussi C, Harris JA, Ruzzoli M (2013). Modelling non-invasive brain stimulation in cognitive neuroscience. Neurosci Biobehav Rev 37: 1702-1712.

Nakagawa S, Schielzeth H (2013). A general and simple method for obtaining R2 from generalized linear mixed-effects models. Methods Ecol Evol 4: 133-142.

Nebes RD, Pollock BG, Houck PR, Butters MA, Mulsant BH, Zmuda MD et al (2003). Persistence of cognitive impairment in geriatric patients following antidepressant treatment: a randomized, double-blind clinical trial with nortriptyline and paroxetine. J Psychiatr Res 37: 99-108.

Nitsche MA, Doemkes S, Karakose T, Antal A, Liebetanz D, Lang N et al (2007). Shaping the effects of transcranial direct current stimulation of the human motor cortex. J Neurophysiol 97: 3109-3117.

Nitsche MA, Jaussi W, Liebetanz D, Lang N, Tergau F, Paulus W (2004). Consolidation of human motor cortical neuroplasticity by d-cycloserine. Neuropsychopharmacology 29: 1573-1578.

Nitsche MA, Kuo MF, Karrasch R, Wachter B, Liebetanz D, Paulus W (2009). Serotonin affects transcranial direct currentinduced neuroplasticity in humans. Biol Psychiatry 66: 503-508. 
Oldfield RC (1971). The assessment and analysis of handedness: the Edinburgh inventory. Neuropsychologia 9: 97-113.

Outhred T, Das P, Felmingham KL, Bryant RA, Nathan PJ, Malhi GS et al (2014). Impact of acute administration of escitalopram on the processing of emotional and neutral images: a randomized crossover fMRI study of healthy women. J Psychiatry Neurosci 39: 267-275.

Poreisz C, Boros K, Antal A, Paulus W (2007). Safety aspects of transcranial direct current stimulation concerning healthy subjects and patients. Brain Res Bull 72: 208-214.

Postma A, Kessels RPC, van Asselen M (2008). How the brain remembers and forgets where things are: the neurocognition of object-location memory. Neurosci Biobehav Rev 32: 1339-1345.

Prehn K, Floel A (2015). Potentials and limits to enhance cognitive functions in healthy and pathological aging by tDCS. Front Cell Neurosci 9: 355.

Reis J, Schambra HM, Cohen LG, Buch ER, Fritsch B, Zarahn E et al (2009). Noninvasive cortical stimulation enhances motor skill acquisition over multiple days through an effect on consolidation. Proc Natl Acad Sci USA 106: 1590-1595.

Savaskan E, Muller SE, Bohringer A, Schulz A, Schachinger H (2008). Antidepressive therapy with escitalopram improves mood, cognitive symptoms, and identity memory for angry faces in elderly depressed patients. Int J Neuropsychopharmacol 11: 381-388.
Shin YI, Foerster Á, Nitsche MA (2015). Transcranial direct current stimulation (tDCS) - application in neuropsychology. Neuropsychologia 69: 154-175.

Siepmann T, Penzlin AI, Kepplinger J, Illigens BM, Weidner K, Reichmann $\mathrm{H}$ et al (2015). Selective serotonin reuptake inhibitors to improve outcome in acute ischemic stroke: possible mechanisms and clinical evidence. Brain Behav 5: e00373.

Sommer T, Rose M, Weiller C, Buchel C (2005). Contributions of occipital, parietal and parahippocampal cortex to encoding of object-location associations. Neuropsychologia 43: 732-743.

Stern RA, Arruda JE, Hooper CR, Wolfner GD, Morey CE (1997). Visual analogue mood scales to measure internal mood state in neurologically impaired patients: description and initial validity evidence. Aphasiology 11: 59-71.

Takahashi E, Ohki K, Kim DS (2008). Dissociated pathways for successful memory retrieval from the human parietal cortex: anatomical and functional connectivity analyses. Cereb Cortex 18: 1771-1778.

van Asselen M, Kessels RPC, Kappelle LJ, Postma A (2008). Categorical and coordinate spatial representations within object-location memory. Cortex 44: 249-256.

Verbeke G, Molenberghs G (2000). Linear Mixed Models for Longitudinal Data. Springer: New York.

Williams E (1949). Experimental designs balanced for the estimation of residual effects of treatments. Aust J Sci Res 2: 149-168.

Supplementary Information accompanies the paper on the Neuropsychopharmacology website. (http://www.nature.com/npp) 